\title{
Brivaracetam: First Canadian Experience in an Intractable Epilepsy Population
}

\author{
Jeanne Lafortune, Charles Deacon, Jean-François Clément
}

\begin{abstract}
Objective: To evaluate the effectiveness and tolerability of brivaracetam (BRV) in a refractory epilepsy population in an outpatient clinical setting. Methods: Retrospective medical information system review and self-report questionnaire for all patients treated with BRV until the end of 2017. Results: Thirty-eight patients were included, $73.7 \%$ female and mean age 36.2 . The mean number of antiepileptic drugs (AEDs) for previous use was 8.9, and for current use was 2.5. Mean seizure frequency in the last 3 months was 12 per month. At 3, 6, 12, and 15 months, the $50 \%$ responder rates were $36.1 \%, 32 \%, 41.2 \%$, and 45.5\%, respectively. Patients took BRV for a median duration of 8.25 months, ranging from 7 days to 60 months. Retention rate was $75.0 \%, 72.0 \%, 59.2 \%$, and $47.9 \%$ at $3,6,12$, and 15 months, respectively. Overall, the main reasons for discontinuation were adverse events (AEs) $(52.3 \%)$, lack of efficacy (35.3\%), or both $(11.8 \%)$. The rate of total AEs was $60.5 \%$ according to medical records and $85.7 \%$ according to questionnaire, including mostly tiredness, psychiatric, and memory complaints. Psychiatric side effects occurred in $31.6 \%$ according to medical records and $47.4 \%$ according to questionnaire results, which is higher than previously reported and persisted throughout the study period. Conclusions: BRV appears to be a useful and safe add-on treatment, even in a very refractory group of patients. In this real-life clinical setting, psychiatric AEs were found at a higher rate than previously published.
\end{abstract}

RÉSUMÉ: Première expérience canadienne avec le brivaracetam comme anticonvulsivant chez des patients avec épilepsie réfractaire. Objectif: Évaluer l'efficacité et la tolérabilité du brivaracétam (BRV) en traitement de l'épilepsie réfractaire dans un contexte de pratique clinique. Méthodes: Procéder à une analyse rétrospective de dossiers médicaux informatisés et faire remplir un questionnaire auto-administré à tous les patients traités par BRV jusqu'à la fin de 2017. Résultats: 38 patients ont été inclus, dont étaient 73.7\% des femmes. La moyenne d'âge était de 36.2 ans. Le nombre moyen de médicaments anticonvulsivants (MAC) utilisés antérieurement était de 8.9 et utilisés au moment de l'étude était de 2.5. La moyenne de crise mensuelle dans les 3 derniers mois était de 12. À 3, 6, 12 et 15 mois, le taux de répondeur 50\% était de 36.1\%, 32\%, 41.2\% et 45.5\%, respectivement. La durée moyenne du traitement par BRV était de 8.25 mois, avec un intervalle de 7 à 60 mois. Le taux de rétention était de 75.0\%, 72.0\%, 59.2\% et $47.9 \%$ à 3,6 , 12 et 15 mois, respectivement. Les principales raisons d'arrêt du BRV étaient en raison d'événements indésirables (EI) (52.3\%), manque d'efficacité (35.3\%) ou les deux (11.8\%). Le taux général d'EI a été de $60.5 \%$ selon les dossiers médicaux informatisés et de $85.7 \%$ selon le questionnaire, incluant surtout de la fatigue ainsi que des plaintes psychiatriques et cognitives. Des EI psychiatriques sont survenus chez $31.6 \%$ des patients selon les dossiers médicaux informatisés et $47.4 \%$ selon le questionnaire, ce qui est plus élevé que précédemment rapporté et a persisté pendant la période de l'étude. Conclusions: BRV apparaît être utile et sécuritaire comme traitement d'appoint, même dans un groupe de patients avec épilepsie très réfractaire. Dans ce contexte concret de pratique clinique, les EI psychiatriques ont été observé plus fréquemment que ce qui a été évoqué antérieurement dans la littérature scientifique.

Keywords: Brivaracetam, Refractory epilepsy, Antiepileptics, Efficacy, Side effects

doi:10.1017/cjn.2019.321

Can J Neurol Sci. 2020; 47: 183-188

\section{INTRODUCTION}

Epilepsy is one of the most prevalent neurological disorders, affecting 40 million people worldwide. ${ }^{1}$ Despite the addition of 15 new anticonvulsants in the past few years, epilepsy remains refractory in $20-30 \%$ of patients. ${ }^{2}$ Moreover, adverse events (AEs) from the use of antiepileptic drugs (AEDs) contribute to treatment failure in up to $40 \%$ of patients and have a considerable impact on quality of life. ${ }^{3}$ Therefore, the development of new therapeutic options for patients with epilepsy is clearly needed.
Brivaracetam (BRV) is the latest AED approved for focal epilepsy and is characterized by its high affinity for selectively binding to synaptic vesicule protein 2A (SV2A), 15- to 30-fold than its analogue levetiracetam (LEV). ${ }^{4} \mathrm{LEV}$ is known to cause psychiatric side effects in $15-30 \%$ of patients, ${ }^{5,6}$ presumably by its negative modulating effect on AMPA receptors. ${ }^{5}$ BRV does not share inhibitory activity on high-voltage $\mathrm{Ca}^{2+}$ channels and AMPA receptors, which could explain a lower incidence of psychiatric AEs than with LEV. ${ }^{4}$ Randomized controlled studies demonstrated that BRV can successfully reduce seizure

From the Department of Neurology, Centre Hospitalier Universitaire de Sherbrooke (CHUS), Sherbrooke, Quebec, Canada (JL, CD, JFC)

Received December 17, 2018. Final Revisions Submitted October 24, 2019. Date of Acceptance October 24, 2019.

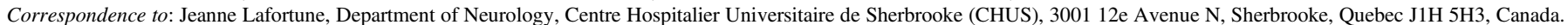
Email: jeanne.lafortune@usherbrooke.ca 
frequency. ${ }^{7-10}$ According to a meta-analysis, the most common AEs associated with BRV are irritability, fatigue, somnolence, and dizziness. ${ }^{11}$ In the largest study, psychiatric AEs occurred in $10.8 \%$ of patients, measured by open-ended questions. ${ }^{8}$

A major limitation when interpreting these pivotal trials is that they do not reflect day-to-day clinical practice. Specifically, these trials have strict entry criteria that exclude many patients with refractory epilepsy or intellectual disability, which constitute an important group of patients in tertiary epilepsy clinics. Therefore, it is important to assess the safety and efficacy in this sub-group. Moreover, phase III trials are limited by their short duration and lack of dosing flexibility. Here, we report post-marketing experience of BRV treatment in our very refractory epilepsy population patients.

\section{Method}

\section{Study Design}

This study consisted of a retrospective, two-center study and was approved by the ethics review board of the Centre Hospitalier Universitaire de Sherbrooke (CHUS). Participants were enrolled in the study between March 2016 and December 2017, except for two patients who initiated treatment as part of a clinical study in 2012. The primary study objective was to assess the effectiveness and tolerability of BRV at different time points $(3,6,12$, and 15 months).

\section{Participants}

The study population comprised all patients who received at least one dose of BRV at the CHUS epilepsy outpatient clinic (tertiary health care center) and Neuro Rive-Sud clinic (a specialized neurology clinic affiliated with the CHUS). Patients with an additional diagnosis of non-epileptic seizures or a recent epilepsy surgery were excluded from efficacy analysis. The 3-month period prior to the introduction of BRV was defined as the baseline. Patients were monitored at the clinician's discretion, with most visits scheduled every 3 months. At every time point, the following items were recorded: number of seizures since the previous visit, changes in medication, and AEs experienced since the previous visit. Reason for discontinuation of BRV (no efficacy, AEs, or both) was noted for each participant.

\section{Data Collection}

All pertinent participant information was collected from the patients' medical files and included age, gender, neuropsychiatric profile, intellectual disability, time since epilepsy onset, seizure type, etiology, seizure frequency in the last 3 months, prior and concomitant AEDs, and prior epilepsy surgery or vagus nerve stimulation. AEs and psychiatric comorbidities were assessed using a short clinical questionnaire completed by the patients or caregivers, adapted from the well-known Liverpool Adverse Events Profile (LAEP-Q), developed in the United Kingdom by the Liverpool Group ${ }^{12}$ and translated to French. The revised version of LAEP-Q was approved by the Liverpool Group and then translated using the BRV monograph ${ }^{13}$ for precise terms.

The efficacy of treatment was calculated using data collected from the medical files. Efficacy outcomes were evaluated by comparing mean number of seizures per month at each visit to the mean number of seizures per month at baseline. Outcomes were defined as $50 \%$ responder rate (responders, including seizure-free patients), minimal responders (0-50\% reduction), and worsening. Seizure freedom was defined as no seizure since the last visit.

AEs were derived from medical records and modified LAEP$\mathrm{Q}$ at two time points: shortly after treatment initiation and at last follow-up for those still under treatment. In the questionnaire, a four-point scale (4 - always or often a problem, 3 - sometimes a problem, 2 - rarely a problem, 1 - never a problem) was used and subsequently dichotomized in $\operatorname{AEs}(4,3)$ or no $\operatorname{AE}(2,1)$ to facilitate result interpretation. In the medical files, the same dichotomization was used (AEs or no AE). AEs were separated into non-psychiatric AEs and psychiatric AEs, including restlessness, anger, ${ }^{1}$ nervousness, depression, emotional lability, irritability, ${ }^{1}$ hallucinations, paranoia, and depersonalization.

\section{Data Analysis}

Statistical analysis was performed using SPSS Statistics v24 (IBM Corp, 2016, Armonk, NY). The Fisher exact test was used with $p$-values $<0.05$ treated as statistically significant for between-group comparison of dichotomous variables. Retention rate was calculated for each assessment point using KaplanMeier estimate. Maintenance doses of BRV were recorded.

\section{RESULTS}

\section{Patients Characteristics at Baseline}

A total of 38 patients $(73.7 \%$ female, mean age 36.2$)$ were included in the analyses of baseline characteristics. Demographic information is summarized in Table 1 . The majority of patients (84.2\%) were diagnosed with focal epilepsy, and the mean epilepsy duration was 22.6 years. More than one-third of patients were intellectually disabled (36.8\%) and more than a half had comorbid mental health issues $(57.9 \%)$. On average, the participants had previously used 8.9 AEDs, $68.4 \%$ of them tried $\geq 8$ different AEDs. At study initiation, patients were taking 2.5 AEDs on average, including BRV. Nearly $90 \%$ of patients had been previously exposed to levetiracetam. The most commonly prescribed drugs at baseline were clobazam (53\%), carbamazepine $(37 \%)$, lamotrigine $(29 \%)$, clonazepam $(26 \%)$, lacosamide $(18 \%)$, and valproate $(16 \%)$. A third of patients underwent unsuccessful epilepsy surgery, and $15.8 \%$ had vagus nerve stimulation therapy. The mean seizure count at baseline was 12 per month.

\section{Efficacy Analysis}

Of the 38 patients in the study, two were excluded from efficacy analysis (recent epilepsy surgery, $n=1$; additional diagnosis of non-epileptic seizures, $n=1$ ). Figure 1 demonstrates the outcomes in terms of $50 \%$ responder rate and number of subjects remaining on efficacy analysis at each assessment time. At 3 months, $36.1 \%(13 / 36$, as per intention-to-treat analysis considering all patients who started BRV) reported at least $50 \%$ reduction in seizures, and five participants were seizure-free

\footnotetext{
${ }^{1}$ Anger is defined as a strong feeling of displeasure, while irritability is defined as a less severe state of quick excitability to annoyance. ${ }^{14}$
} 
Table 1: Epidemiological and epilepsy data

\begin{tabular}{|c|c|c|}
\hline & $n(\%)$ & Mean (SD) \\
\hline Total number of patients & 38 & \\
\hline Age & & $36.2(13)$ \\
\hline Gender (woman/men) & $28 / 1073.7 \%$ female & \\
\hline $\begin{array}{l}\text { Time since epilepsy onset } \\
\text { (years) }\end{array}$ & & $22.6(13)$ \\
\hline \multicolumn{3}{|l|}{ Seizure onset, $n(\%)$} \\
\hline Focal & $32(84.2)$ & \\
\hline Primary generalized & $6(15.8)$ & \\
\hline \multicolumn{3}{|l|}{ Etiology, $n(\%)$} \\
\hline Genetic & $14(36.8)$ & \\
\hline Structural & $13(34.2)$ & \\
\hline Immune & $1(2.6)$ & \\
\hline Unknown & $10(26.3)$ & \\
\hline $\begin{array}{l}\text { Seizure frequency } \\
\text { (per month) }\end{array}$ & & $12.4(12)$ \\
\hline No. of previous AEDs & & $8.9(3)$ \\
\hline $0-3$ & 0 & \\
\hline $4-7$ & $12(31.6)$ & \\
\hline$\geq 8$ & $26(68.4)$ & \\
\hline No. of concomitants AEDs & & $2.5(1.08)$ \\
\hline 1 & $6(15.8)$ & \\
\hline 2 & $12(31.6)$ & \\
\hline 3 & $11(28.9)$ & \\
\hline 4 & $8(21.1)$ & \\
\hline Prior epilepsy surgery & $12(31.6)$ & \\
\hline Vagus nerve stimulation & $6(15.8)$ & \\
\hline Neuropsychiatric profile & $22(57.9)$ & \\
\hline Anxiety & $18(47.4)$ & \\
\hline Depression & $12(31.6)$ & \\
\hline Psychosis & $1(2.6)$ & \\
\hline Intellectual disability, $n(\%)$ & $14(36.8)$ & \\
\hline
\end{tabular}

(13.4\%). There was minimal response in three patients $(8.3 \%)$, no change in six patients (16.6\%), and an increase in seizure frequency was observed in six patients $(16.6 \%)$. At 6 months, $32 \%(8 / 25$, again as per intention-to-treat analysis) of patients were responders, and four of them were seizure-free. Two $(8 \%)$ had $<50 \%$ responder rate, while six $(24 \%)$ reported no change, and seven $(28 \%)$ had an increase in seizures. Long-term response of at least $50 \%$ was apparent in $41.2 \%$ of patients $(7 / 17$, two patients were seizure-free) at 12 months, and $45.5 \%$ (5/11, two patients were seizure-free) at 15 months. In one patient, the response was unknown at 15 months. No statistically significant differences in efficacy outcomes were observed between focal epilepsy and generalized epilepsy syndromes $(p=0.33)$.

\section{Adverse Events}

AEs captured by the self-report questionnaire were more frequent than those found in medical records.

\section{a. Questionnaire}

There was a $73.7 \%$ response rate for the self-report questionnaire $(n=28)$. Initial AEs occurred in $85.7 \%$ of patients. The most frequent AEs were tiredness (39.5\%), memory problems $(34.2 \%)$, sleepiness $(31.6 \%)$, and restlessness (31.6\%). Total psychiatric side effects occurred in $47.4 \%$, including anger in $23.7 \%$, nervousness in $21.1 \%$, depression in $23.7 \%$, emotional lability in $28.9 \%$, irritability in $26.3 \%$, hallucinations in $7.9 \%$, paranoia in $10.5 \%$, and depersonalization in $5.3 \%$; $28.9 \%$ of patients had dizziness. At the last follow-up, $81.0 \%$ of patients who remained on BRV experienced AEs, including $34.2 \%$ of patients who still had psychiatric AEs. Most AEs reported at follow-up were similar to those first reported after starting BRV. Questionnaire response rate was $100 \%$ for those who remained on BRV at the last follow-up.

\section{b. Medical records}

According to medical records, initial AEs occurred in $60.5 \%$ of patients. The most frequent were tiredness $(21.1 \%)$, emotional lability (15.8\%), nervousness (13.2\%), and sleepiness (13.2\%). Total psychiatric side effects occurred in $31.6 \%$, including restlessness in $5.3 \%$, anger in $7.9 \%$, depression in $7.9 \%$, irritability in $10.5 \%$, hallucinations in $5.3 \%$, paranoia in $5.3 \%$, and depersonalization in $5.3 \%$. Only $7.9 \%$ of patients reported dizziness. At the last follow-up, $28.6 \%$ of patients who remained on BRV experienced AEs, and similar to the questionnaire, emotional lability and nervousness were most frequently experienced, and depression and irritability were also notable. In total, $23.8 \%$ of patients still had psychiatric AEs at the time of the last follow-up.

Two patients discontinued BRV $<1$ week because of intolerable AEs: one experienced hand paresthesia, and the other suffered from intense pruritus without visible skin rash. Major AEs occurred in two patients taking BRV. One suffered from traumatic cervical cord injury after a seizure-related fall, and another developed status epilepticus within 1 week of beginning BRV. There was a trend suggesting that psychiatric AEs were more common among patients with psychiatric comorbidity ( $40.9 \%$ vs $18.8 \%, p=0.178)$. Patients with intellectual disability were not more prone to experiencing psychiatric AEs $(35.7 \%$ vs $29.2 \%, p=0.675)$. One patient without a history of psychiatric illness developed frank psychosis 4 weeks into treatment, with religious delusions, visual and auditory hallucinations, and paranoia. Following BRV cessation, those symptoms resolved completely. Among patients who continued the drug, $42.9 \%$ felt unsatisfied with the treatment, according to the questionnaire.

\section{Dosage}

Mean daily BRV dosage was $144 \mathrm{mg}$ (SD 52). Patients who discontinued the medication took a mean daily dose of $117.5 \mathrm{mg}$ (SD 55). Patients who became seizure-free between each time point took a mean daily dose of $161.54 \mathrm{mg}$ (SD 45.2). Four patients remained seizure-free throughout the study period and were taking BRV for 7 months $(50 \mathrm{mg})$, 9 months $(200 \mathrm{mg}$ ), 12 months (200 mg), and 15 months (100 mg).

\section{Retention Rate}

Patients in the study completed BRV therapy for a median duration of 8.25 months, ranging from 7 days to 60 months. 

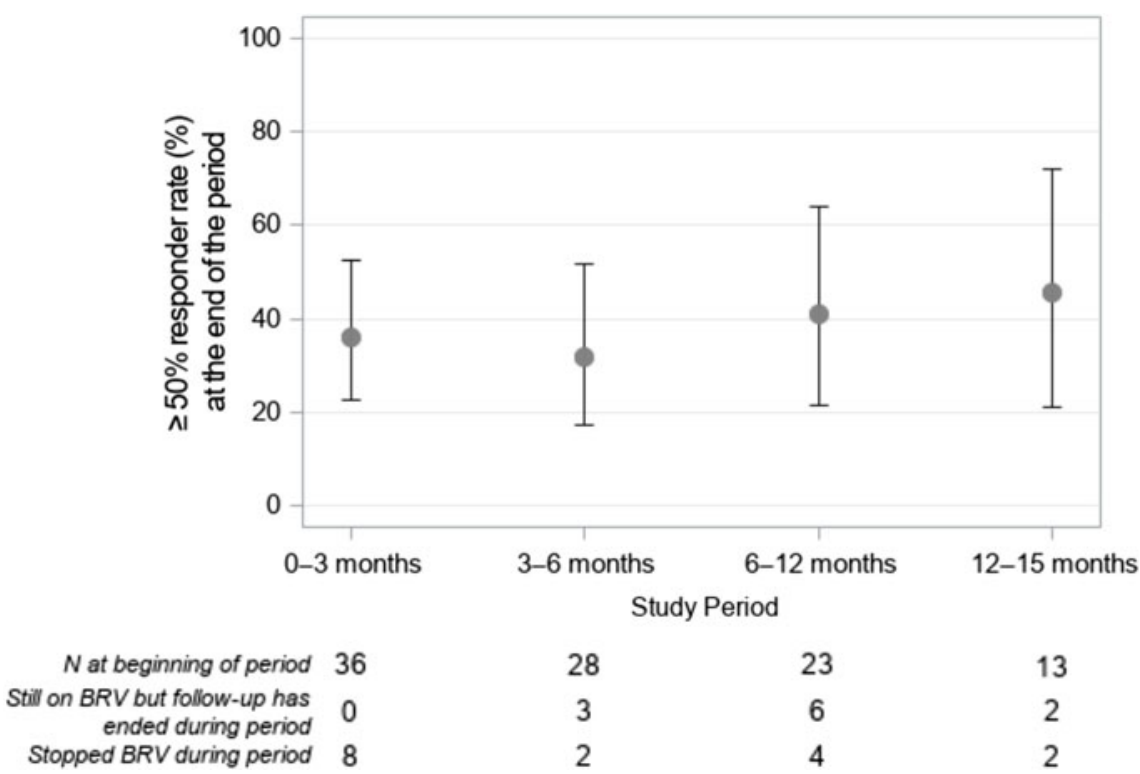

Figure 1: 50\% responder rate at 3-, 6-, 12-, and 15-month follow-up visits and number of subjects remaining on efficacy analysis at each assessment time point.

Retention rate calculated using the Kaplan-Meier survival curve from the beginning of exposure was $75.0 \%, 72.0 \%, 59.2 \%$, and $47.9 \%$ at $3,6,12$, and 15 months. Overall, $44.4 \%$ of patients discontinued BRV during the study period. Considering only those who discontinued, median time from BRV start to discontinuation was 3 months, ranging from 7 days to 60 months. Reasons for discontinuation were AEs in 52.3\% $(n=9)$, lack of efficacy in $35.3 \%(n=6)$, and both in $11.8 \%(n=2) ; 54.5 \%$ of patients who discontinued treatment due to AEs did so because of psychiatric AEs. Of note, two patients received the drug for approximately 60 months, following inclusion in a clinical trial in 2012. One of them discontinued after 59 months because of irritability (unclear if related to BRV); this participant was then switched to lacosamide without much improvement. The other patient remained on BRV treatment at the final assessment point and was seizure-free.

\section{DISCUSSION}

This retrospective real-world study reflects the use of BRV in daily clinical practice as an add-on AED in a very challenging epilepsy population. Indeed, our sample included a high proportion of intellectually disabled patients, and almost a third had undergone unsuccessful epilepsy surgery. Patients had tried a mean number of 8.9 AEDs, and $50 \%$ were currently taking $\geq 3$ AEDs.

Comparing the patients of present cohort with those of earlier trials, the responder rate was similar $(36.1 \%(23 / 36)$ at 3 months in the present study and $27.3-55.8 \%$ in earlier trials ${ }^{7-10,15-17}$ ). Recently, five clinical studies ${ }^{18-22}$ from Germany and one from Spain $^{23}$ assessed seizure control and tolerability using a similar study design. These trials also showed $50 \%$ responder rates ranging from $27.8 \%$ to $45 \%$ at 3 months. Considering that patients at the beginning of those studies were being treated with a mean number of 2.23 AEDs and that the mean number of unsuccessfully tried AEDs was 5.9, it is likely that the cohort of the present study is more or at least as refractory as that of other post-marketing clinical studies. Responder rate at 6 and 12 months was also in line with other BRV post-marketing studies. $^{19,21-23}$

In our study, the rate of seizure freedom was $5 / 36(13.4 \%)$, $4 / 25$ (8\%), 2/17 (11.8\%), and $2 / 11(18.2 \%)$ at 3, 6, 12, and 15 months, respectively. These rates are similar to those reported in previous studies (ranging from $7 \%$ to $25 \%$ ). $^{18-23}$

The occurrence of initial AEs was higher $(60.5 \%$ according to medical records and $85.7 \%$ according to questionnaire data) compared to other retrospective clinical series $(16-39.8 \%) .{ }^{18-23}$ Importantly, no other retrospective clinical series have used a self-report questionnaire, possibly in part explaining the discrepancy between those results. Our AE rates are in line with those of the pooled analysis of placebo-controlled studies $(68.3 \%)^{13}$ and pooled data from long-term follow-up studies, which showed that AEs are reported by $84.5 \%$ of patients and considered to be treatment-related by $54.2 \% .^{16}$ In analyzing these results, one has to bear in mind the considerable drug load of the present study population and that the incidence of AEs increases with the number of drugs used. The most frequently reported initial AEs in our study were tiredness, memory problems, and restlessness based on the self-report questionnaire, in contrast to tiredness, nervousness, and emotional lability based on medical records. At the last followup of those who were maintained on BRV treatment, tiredness, memory problems, and psychiatric side effects (emotional lability, depression, irritability, nervousness) were a common complaint. The incidence of memory difficulties reported by patients in the questionnaire was high $(34.2 \%)$ compared with $1.9 \%$ in a previous study by Steinig et al., ${ }^{19} 4.7 \%$ by Villanueva et al., ${ }^{23}$ and $1-2 \%$ in pivotal clinical trials. ${ }^{7-10}$ This discrepancy could again be explained by the over-reporting of AEs in a questionnaire (in contrast to the open-ended questions used in pivotal clinical trials), ${ }^{24}$ by the different population characteristics and cultural perception or could simply be a spurious finding.

Initial psychiatric side effects occurred in $31.6 \%$ of our patients according to medical records and $47.4 \%$ according to questionnaire data, compared to $10.8 \%$ in the Klein et al. study ${ }^{8}$ 
and ranging from $14 \%$ to $22.6 \%$ in clinical studies. ${ }^{19-23}$ To date, no studies directly comparing BRV with LEV have been published. However, in our study, psychiatric side effects were more frequent with BRV therapy than that described in other studies with LEV therapy $(15-30 \%) .^{5-6}$ This suggests that psychiatric AEs may be higher with BRV than it was previously demonstrated in clinical trials. Our study also contrasts with the study by Yates et al., ${ }^{25}$ a small open-label prospective trial where 29 patients experiencing psychiatric AEs with LEV were switched to BRV. After 3 months, $>90 \%$ of patients experienced a clinically meaningful reduction of those AEs, suggesting that patients experiencing psychiatric AEs with LEV use could benefit from a switch to BRV. Villanueva et al. (2018) also reported improved tolerability profile in patients transitioned from LEV to BRV. ${ }^{23}$

Retention rates of $75 \%$ at 3 months, $72 \%$ at 6 months, and $53.2 \%$ at 1 year are comparable to other series using BRV $^{18-19,21-23}$ and also other AEDs licensed for focal epilepsies. $^{20}$ In those who continued the drug, $42.9 \%$ felt unsatisfied with the treatment. This high number could reflect the persistence of side effects or simply the persistence of seizures despite a new medication.

\section{Limitations}

However, certain limitations have to be considered. The current study used a retrospective design, which is intrinsically linked to a risk of $\mathrm{AE}$ underestimation. Conversely, the use of a self-report questionnaire for measuring patient-reported side effects of drugs may have overestimated AEs. Also, the questionnaires were filled in by patients long after the introduction of medication, which could have brought in recall bias, an intrinsic limitation of studies using self-reported data. ${ }^{24}$ The concomitant use of a questionnaire and data from medical records may have lessened the impact of this issue. A similar reflection applies to seizure frequency assessment. Recent studies suggest that seizure characteristics reported by patients and caregivers are of poor quality, ${ }^{26}$ especially for patients with a high number of daily seizures or seizure clusters. This is an inherent limitation of selfreported seizure frequency and can be improved by using a seizure diary. However, no formal seizure diary was used by the clinicians in our study. Our cohort also included patients with intellectual disabilities, which caused difficulty in using a selfreported diary as a standardized method of collecting data. In addition, many symptoms listed in the modified LAEP-Q, such as depression or memory problems, are also extremely common comorbidities in the epilepsy population. Therefore, it is challenging to know whether they are attributable to the medication or a comorbid state of pre-existing epilepsy and, if so, to measure the impact of each. Finally, the small sample size may limit the external validity of those results.

\section{Strengths}

The added value of the present study mainly lies in the fact that it includes the understudied subgroup of very refractory epilepsy patients and an overall long-term follow-up time (up to 60 months). Moreover, it assessed drug efficacy in a real-world setting using both medical records and a validated questionnaire. Additionally, the use of a self-report questionnaire allows patients to really communicate what they experience with new therapy, possibly more freely than with the traditional interview. The risk of selection bias was also low by way of including all patients who received BRV as of the end of 2017 (predefined time).

\section{Conclusion}

The addition of BRV to a regimen of AEDs in patients with refractory epilepsy appears to be effective and safe in real-life conditions. Ease of use and a favorable pharmacokinetic profile make this drug an appealing choice as an add-on AED. However, in our cohort, psychiatric AEs were found at a higher rate than previously published. Self-report questionnaire may be used to help identify AEs that are missed in traditional medical records, such as neuropsychiatric AEs.

\section{ACKNOWLEDGMENTS}

The authors thank Joanna Wai Ling Ma, BScN, for her help in collecting questionnaire data, and Marie-Pierre Garant for her help with statistical analysis. The authors also thank Samantha Côté and Christian Bocti for their useful comments on the manuscript. This study was not supported by any external sources.

\section{STATEMENT OF AUTHORSHIP}

JL and CD conceived the original idea. JL did collection, analysis, and interpretation of data, as well as writing and revising of the manuscript. CD and JFC read, provided comments, and approved the final version of the article.

\section{Disclosures}

The authors have no conflict of interest to declare.

\section{REFERENCES}

1. Programme de recherche sur la génétique des épilepsie, Statistiques, CHUM centre de recherche/université de Montréal, www.epileptogene.ca/

2. Leoscher W, Schmidt D. Modern antiepileptic drug development has failed to deliver: ways out of the current dilemma. Epilepsia. 2011;52:657-78.

3. Perucca E, Meador KJ. Adverse effects of antiepileptic drugs. Acta Neurol Scand Suppl. 2005;181:30-5.

4. Leoscher W, Gillard M, Sands ZA, Kaminski RM, Klitgaard H. Synaptic vesicle glycoprotein $2 \mathrm{~A}$ ligands in the treatment of epilepsy and beyond. CNS Drugs. 2016;30:1055-77.

5. Hansen C, Ljung H, Brodtkorb E, Reimers A. Mechanisms underlying aggressive behavior induced by antiepileptic drugs: focus on topiramate, levetiracetam, and perampanel. Behav Neurol. 2018;2018, Article ID 2064027, 18 p.

6. Helmstaedter C, Mihov Y, Toliat MR, et al. Genetic variation in dopaminergic activity is associated with the risk for psychiatric side effects of levetiracetam. Epilepsia. 2013;54(1):36-44.

7. Ryvlin P, Werhahn KJ, Blaszczyk B, et al. Adjunctive brivaracetam in adults with uncontrolled focal epilepsy: results from a double-blind, randomized, placebo-controlled trial. Epilepsia. 2014;55:47-56.

8. Klein P, Schiemann J, Sperling M, et al. A randomized, doubleblind, placebo-controlled, multicenter, parallel-group study to evaluate the efficacy and safety of adjunctive brivaracetam in adult patients with uncontrolled partial-onset seizures. Epilepsia. 2015;56:1890-8.

9. Biton V, Berkovic S, Abou-Khalil B, Sperling M, Johnson M, Lu S. Brivaracetam as adjunctive treatment for uncontrolled partial epilepsy in adults: a phase III randomized, double-blind, placebo-controlled trial. Epilepsia. 2014;55:57-66. 
10. Kwan P, Trinka E, Van Paesschen W, Rektor I, Johnson M, Lu S. Adjunctive brivaracetam for uncontrolled focal and generalized epilepsies: results of a phase III, double-blind, randomized, placebo-controlled, flexible-dose trial. Epilepsia. 2014;55:38-46.

11. Lattanzi S, Cagnett C, Foschi N, Provinciali L, Silvestrini M. Brivaracetam add-on for refractory focal epilepsy: a systematic review and meta-analysis. Neurology. 2014;86:1344-52.

12. Baker GA, Frances P, Middleton E. Initial development, reliability and validity of a patient-based adverse events scale. Epilepsia. 1994; 35;80.

13. UCB Canada Inc. BrivleraTM (brivaracetam): Canadian product monograph. 2016. Available at: https://s3.pgkb.org/attachment/ brivaracetam_HCSC_Aug2016.pdf; accessed 11 November 2019.

14. "Anger" and "Irritability". Merriam-Webster.com. MerriamWebster 2019. Web. Accessed March 5, 2019.

15. French JA, Costantini C, Brodsky A, Von Rosenstiel P, N01193 Study Group. Adjunctive Brivaracetam for refractory partialonset seizures: a randomized, controlled trial. Neurology. 2010;75(6):519-25.

16. Toledo M, Whitesides J, Schiemann J, et al. Safety, tolerability, and seizure control during long-term treatment with adjunctive Brivaracetam for partial-onset seizures. Epilepsia. 2016; 57(7):1139-51.

17. Van Paesschen W, Hirsch E, Johnson M, Falter U, von Rosenstiel P. Efficacy and tolerability of adjunctive Brivaracetam in adults with uncontrolled partial-onset seizures: a phase IIb, randomized, controlled trial. Epilepsia. 2013;54(1):89-97.

18. Steinhoff BJ, Bacher M, Bucurenciu I, Hillenbrand B, Intravooth T, Kornmeier R, et al. Real-life experience with Brivaracetam in 101 patients with difficult-to-treat epilepsy- a monocenter survey. Seizure. 2017;48:11-4.

19. Steinig I, von Podewils F, Moddel G, et al. Postmarketing experience with Brivaracetam in the treatment of epilepsies: a multicenter cohort study from Germany. Epilepsia. 2017; 58(7):1208-16.

20. Zahnert F, Krause K, Immisch I, et al. brivaracetam in the treatment of patients with epilepsy-first clinical experiences. Front Neurol 2018;9:38.

21. Willems LM, Bertsche A, Bösebeck F, et al. Efficacy, retention, and tolerability of brivaracetam in patients with epileptic encephalopathies: a multicenter cohort study from Germany. Front Neurol 2018;9:569.

22. Strzelczyk A, Kay L, Bauer S, et al. Use of brivaracetam in genetic generalized epilepsies and for acute, intravenous treatment of absence status epilepticus. Epilepsia. 2018;59:1549-56.

23. Villanueva V, Lopez-Gonzalez FJ, Mauri JA, et al. BRIVA-LIFE-A multicenter retrospective study of the long-term use of brivaracetam in clinical practice. Acta Neurol Scand. 2019;139: 360-8.

24. Althubaiti A. Information bias in health research: definition, pitfalls, and adjustment methods. J Multidiscip Healthc. 2016; 9:211-7.

25. Yates SL, Fakhoury T, Liang W, Eckhardt K, Borghs S, D’Souza J. An open-label, prospective, exploratory study of patients with epilepsy switching from levetiracetam to brivaracetam. Epilepsy Behav. 2015;52:165-8.

26. Fisher R, Blum DE, DiVentura B, et al. Seizure diaries for clinical research and practice: limitations and future prospects. Epilepsy Behav. 2012;24:304-10. 